\title{
Research on Simulation of Grid Communication Network
}

\author{
CAO Lei \\ Command information system college \\ PLA University of Science and Technology \\ NanJing, China \\ ZHANG Huiwen \\ Command information system college \\ PLA University of Science and Technology \\ NanJing, China
}

\author{
LV Zonghang \\ Command Information System College \\ PLA University of Science and Technology \\ NanJing, China \\ Lvzonghang321@163.com \\ CHEN Xiliang \\ Command information system college \\ PLA University of Science and Technology \\ NanJing, China
}

\begin{abstract}
The construction of grid communication network is a complicated and time-consuming work. So, we offer a simulation model of grid communication network in order to meet the needs of the experiments and research. In this paper we analyze the features and architecture of grid communication network, study the key technologies of grid communication simulation deeply, propose a simulation structure model for grid communication network, and build a prototype simulation system.
\end{abstract}

Keywords- grid, grid communication network, simulation

\section{INTRODUCTION}

Grid communication network is the basic network carrying environment for Global Information Grid, as in [1], the realization of the target capability of the Global Information Grid depends on the construction of the grid communication network. However, due to the wide distribution of heterogeneous networks and technical limitations of grid services, the realization of grid communication network must be a lengthy and complicated process. In order to explore the idea of grid communication network construction to meet the needs of experiments and research, we study how to construct a simulation grid communication environment.

\section{ARCHITECTURE OF GRID COMMUNICATION NETWORK}

Grid communication network is a communication environment, as in [2], realized through grid technique. By providing resources admission control, routing, resource allocation and network management services, it facilitates users to find process and exchange information, and optimizes network resources better.

Grid communication network is not a simple interconnected mesh network but a communication network based on SOA which supports grid applications better. Heterogeneous communication network resources are its foundation. By using the technique of service encapsulation, it offers standard interface for unified communications. It is similar to power grid which unifies all kinds of power resources (thermal power, wind power, hydropower, and nuclear power) to provide users with standard power supply interface and standard electrical equipments plug-andplay. Grid communication network is a comprehensive interconnected communication network with heterogeneous communication networks such as wired, wireless and satellite network. It manages all network resources and provides standard service interface to users with the technology of service-oriented encapsulation. Services and applications which are up to standard can be accessed and use the communication services. Ideally, the grid communication network is a plug-and-play "network". Any application can be inserted into the network no matter when and where, and get the corresponding services.

The layered model of grid communication network based on SOA is shown in Figure 1.

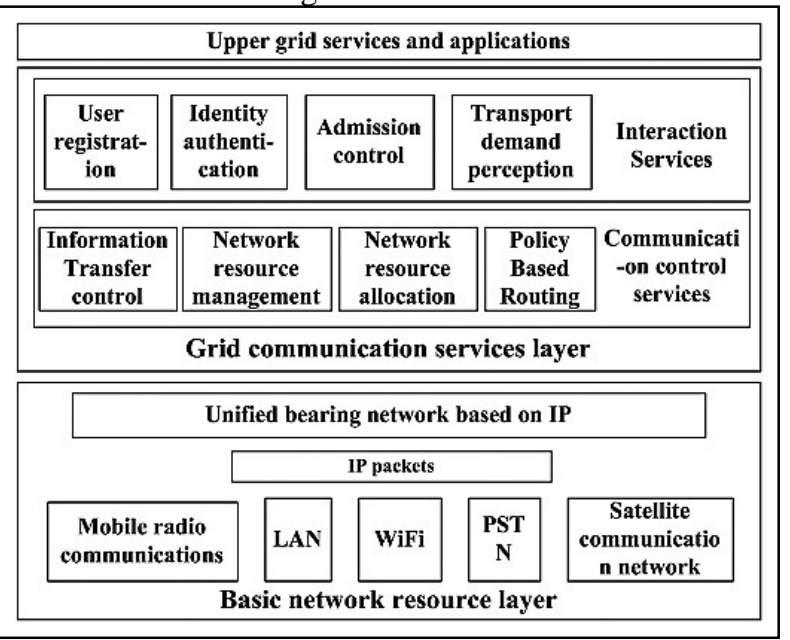

Figure 1. A layered model of grid communication network based on SOA

Through adding grid communication services layer on basic network resources layer, grid communication network based on SOA, as in [3], offers distributed network resources and communication capability together with computing resources and storage resources as services to upper-layer applications. In this way, it integrates communication networks into grid environment better. 
Basic network resources of grid communication network are mainly made up of various heterogeneous communication networks and the internetworking and communication equipments, such as switches and routers, used for network interconnection. They are responsible for transmitting the specific information in grid communication network and are the basis for realizing the functions of a grid communication network.

Grid communication services layer is located above the basic network resources layer. It is a common set of core services which are responsible for realizing the main functions of a grid communication network. In accordance with the function of communication services and their relationship with network resources or upper layer services, grid communication services can be divided into interaction services and communication control services. Communication control services contain information transmission control, network resource management, policybased routing, and network resource allocation. They are services closely related to network and mainly responsible for network management and resource allocation. They send data to network for transmitting. Interaction services, used to interact with upper services, are made up of user registration, ID authentication, admission control and transport demand perception. The communication services layer serves as a connecting link between the preceding and the following by shielding the isomerism details of underlying network for upper layer applications on one hand and providing communication services to meet communication requirements required by upper layer applications on the other.

\section{KEY TECHNOLOGY OF GRID COMMUNICATION NETWORK SIMULATION}

Realization of grid communication network is a complicated project. Many technical problems need to be researched further, including user access technology, transport demand perception technology, admission control technology, information transmission control technology, network resource management technology, and heterogeneous integrated network technology. It is hardly realistic that we study all of these technologies and simulate a complete grid communication network. The purpose of this paper is to explore how to build a basic grid communication environment, our study only focus on the key technologies supporting the simulation of a grid communication network with basic functions.

\section{A. User registration and admission control}

Grid communication network offers "plug and play" to users. As long as a user accesses the network and logs in with a legal user ID normally, a network address can be assigned, and the transport demand can be submitted to grid communication network. Transmission needs of the user can be automatically sensed by transport demand perception service and accepted or rejected by admission control service depending on the admission control strategy. The purpose of admission control service is to balance the contradiction between maximum utilization of resources and user's QoS requirements. In various admission control technologies, policy-based admission control can effectively ensure service quality, safety and reliability for different users or different business, as in [4].

When we design simulation for grid communication network, we adopt an approach for user registration that user's identification is fixed but user's address is assigned when they access to network. Each user that accesses to network has his fixed unique identifier, while the address for a user is allocated according to the location where the user accesses to the network and the equipment being used. The registration information of this user is unified managed by registration information database. The simulation of admission control service is based on the combination of policy-based admission control and transmission demand perception. We design and program a priority-based admission control method. Its workflow is as follows:

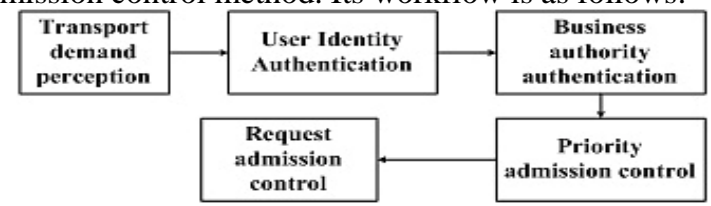

\section{B. Network resource management and transfer control}

The purpose of information transfer control is to dispatch the transport demands that have been accepted reasonably, determine the transmitting rules (method of information transmission, QoS assurance mechanism and priority) for a transport demand in the network, and match the demand with network resource status to achieve the goal of information transmission on-demand. Network resource management provides the description of network resources and the network statuses which are necessary for transmission control and network automatically configuration. As the underlying network of grid communication network is complicated and diverse, we need a more efficient network resource management service. Reference [5] proposed a resource management technology based on QoS and multilayer cooperation, which can combine service access control with routing and channel resource reservation better and optimize network by configuring network according to its conditions more efficiently. So we combine a resource management technology based on QoS and cooperation with the design of admission control for simulation of network resource management and transmission control. Its basic workflow is as follows:

\begin{tabular}{|c|c|c|c|}
\hline & Strategy & Network & Information \\
\hline & reserve & matching & transmission \\
\hline
\end{tabular}

\section{Heterogeneous intergrated network technology}

Functions of grid communication network are realized by network communication services based on the unified network, so the interconnection of the ground heterogeneous network affects the quality of grid communication services directly. With the maturity and popularity of IP business and its associated standards, technologies and infrastructures, we have got a common view that IP network is also the core network as the infrastructure of next generation network. 
Heterogeneous interconnected networks based on IP carrying network can shield the differences of network topology and communication protocols existing in networks, complete networks interconnection and provide ubiquitous information transmission capability for grid communication network, as in [6]. Shown in Figure 2 is an interconnection structure of IP based heterogeneous network.

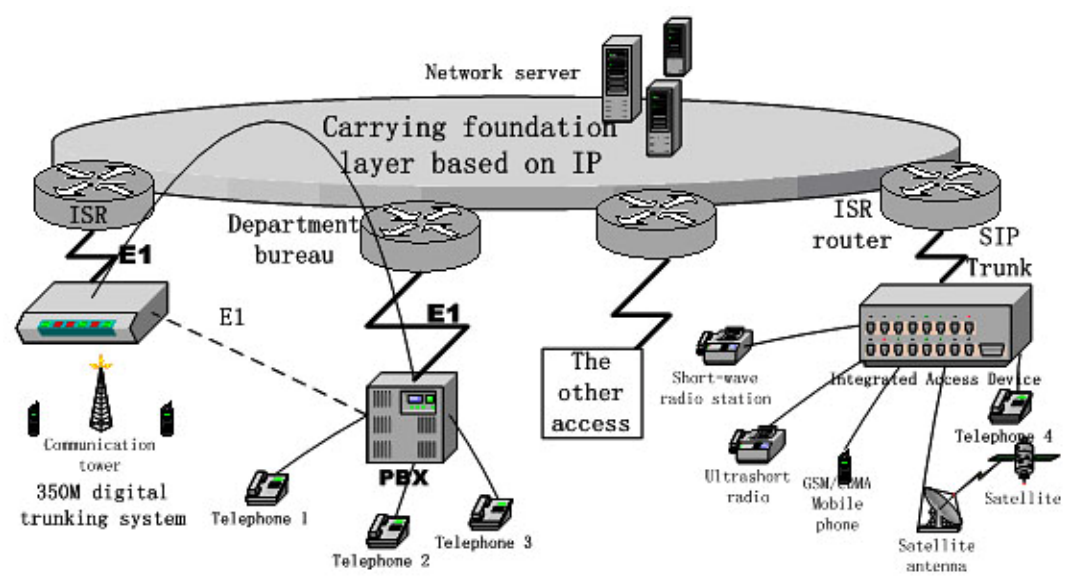

Figure 2. Interconnection structure of IP-based heterogeneous network

\section{Simulation Design OF GRID COMMUNiCATION NETWORK}

\section{A. Simulation model structure}

According to the research on architecture of grid communication network and key simulation technologies, we design a basic simulation model structure for grid communication network, as shown in Figure 3.

This model mainly includes two parts: the bottom is the simulation model of unified network which is established by simulation tool and deployed on simulation servers. The simulation tool we chose can simulate networks realistically through its powerful protocol model. On top of the simulation network is the simulation of grid communication services layer. In this paper, we design the following function modules to simulate the basic functions of communication services according to the key techniques:

- User registration module, which implements the function of user registration and storages user's information in user registration database;

- Transport demand perception module, which perceives user's transport request;

- User authentication module, which Identifies user's verification based on information in registration database;

- Business permission verify module, which is the first step of policy-based admission control, verify business privileges of user's;

- Business accepted module, which is based on priority rules and strategy match;

- Information transfer control module which is based on QoS requirements of business transport and status of network resource.

\section{B. Prototype simulation system of Grid Communication Network}

Development of common network simulation tool improves simulation performance of the base network. QualNet is an integrated platform used to simulate wired and wireless networks of large scale with providing advanced emulation and simulation technology and supporting multiple operating systems. We can use it to simulate real network and complicated large network more precisely with high speed and real time. According to the technology of heterogeneous networks interconnection based on IP, we chose the QualNet simulation tool to build the basic network simulation environment.

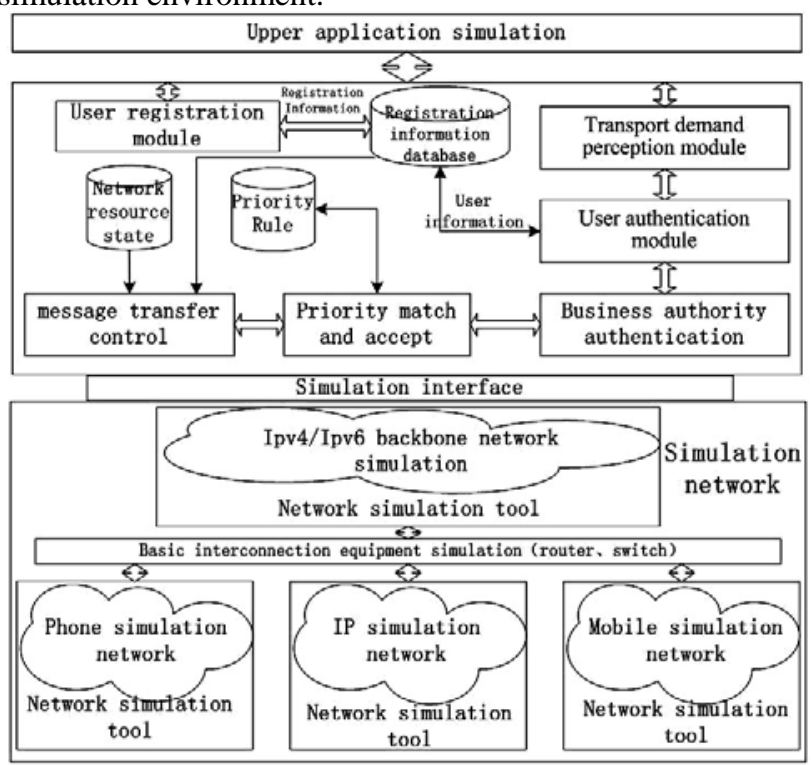

Figure 3. Simulation model structure of grid communication network 


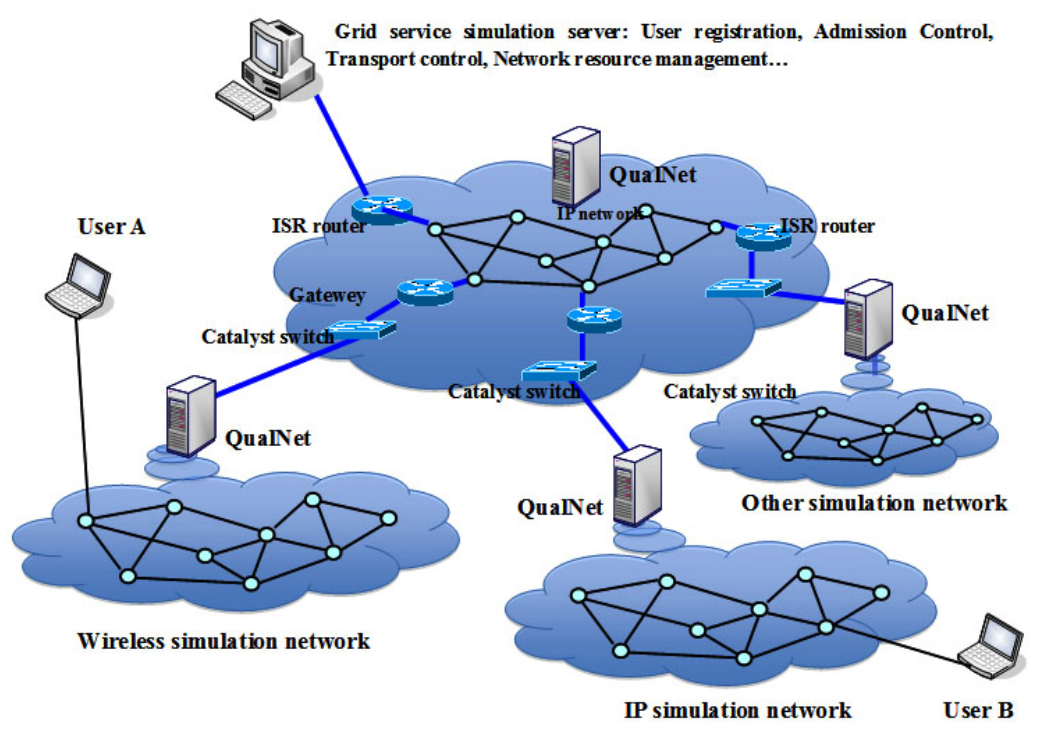

Figure 4. Prototype simulation system of grid communication network

To simulate grid communication services layer, we design a specific communication services emulator and deploy it on a host as simulation server of grid communication services. Our prototype simulation system of grid communication network, as shown in Figure 4, consists of four QualNet network simulation servers and a grid communication services simulation host. The server which simulates basic IP network is the centric of the system with other servers and the service simulation host connecting to it.

\section{Function of simulation system}

The prototype simulation system of grid communication network we design in this paper has the capability of simulating basic functions of grid communication services, including user-to-user information transport and user's getting resources from network. Let's take a look at the userto-user information transport as an example. Its simulation process is as follows:

- a. User A and user B access to network, the registration service assigns address for them and records the position information.

- b. User A submits its information transmission request with a destination of user B's to the grid communication service.

- c. Admission control service identifies request and check identification of the user $\mathrm{A}$, transport request will be received if authentication get through, then admission control service will verify the business rights, and match it with policy.

- d. If business privileges get through, transmission control service will accept the transfer task, according to the result of the priority policy matching, and match the business type and priority with network resource status;

- e. Information transmission function receives the information and transmits it, and queries the registration information of user $B$. Then Information will be transmitted to user $\mathrm{B}$ by the integrated network.

\section{CONCLUSION}

Grid communication network is the basis of grid information system, the simulation of it is of great importance for studying the real network, guiding its construction, and doing experiments on technology research. In this paper we introduce the grid communication network with network functions, architecture and system composition, discuss the key technologies for simulating a grid communication network and design a prototype simulation system. Our research in future will focus on completing the function of the grid communication network simulation system, such as improving the function of services and designing standard interfaces of grid services to support service register discover and invoke.

\section{REFERENCES}

[1] KANG Zhibin, ZHAO Xinguo, HUANG Chenglin, "Research On the Communication Architecture of Global Information Grid,” Journal of the Academy of Equipment Command \& Technology, Vol.17, Feb, 2006, pp.97-101

[2] TANG Ning, YIN Hao, LIU Jin, "Construction and development of Communications Grid in U.S. military," Military electronic information academic conference proceedings, Oct. 2006, pp.588-591

[3] FAN Shuyan, XIONG Gaoyun, "Research on architecture and key technology of the grid communication network," Journal of Xi'an University of Electronic Science and Technology, Vol.36, Dec. 2009, pp. 990-995

[4] YANG Liwei, "Research on the strategy of integrated access system in communication grid," Xi'an University of Electronic and Science, 2010

[5] WANG Peng, "Research on resource management of communication grid,” Computer Engineering, Vol.38, Nov.2012, pp.80-84

[6] CHEN Shouxue, "Network Selection in Heterogeneous Network," Beijing University of Post and Telecommuniations, 2010 